\title{
It May Happen: Endometrioma from Hemorrhagic Corpus Luteum
}

\author{
Juan Piazze ${ }^{1 *}$, Ettore Palma ${ }^{2}$, Francesco Recchia ${ }^{3}$, Cristina Galligani ${ }^{4}$, Silvio Rea ${ }^{5}$ \\ ${ }^{I}$ ASL Frosinone (Poliambulatorio di Ceprano-Ospedale SS Trinità di Sora), Fondazione Carlo \\ Ferri, Monterotondo, Rome \\ ${ }^{2}$ Department of Gynecological Obstetrics and Urologic Sciences of "Sapienza" University of Rome \\ ${ }^{3}$ Ospedale Civile di Avezzano, Fondazione "Carlo Ferri", Monterotondo, Rome \\ ${ }^{4}$ Analisi e Diagnostica Neomedica, Rome \\ ${ }^{5}$ Chirurgia Oncologica, Università degli Studi de L'Aquila, Dpt DISCAB, Fondazione "Carlo Ferri", \\ Monterotondo, Rome
}

*Corresponding Author: Juan Piazze, ASL Frosinone (Poliambulatorio di Ceprano-Ospedale SS Trinità di Sora), Fondazione Carlo Ferri, Monterotondo, Rome, Email: cloudpia@me.com

Abstract: High Doppler resistive indices in a hemorrhagic corpus luteum suggest a possible an eventful transformation to endometrioma.

Keywords: Endometrioma, hemorrhagic corpus luteum, ultrasound.

\section{INTRODUCTION}

The demonstration of a direct transition to endometrioma from a follicle or a hemorrhagic corpus luteum has been described in few studies, it remains the classical theory describing pathogenesis of endometriomas from entrapment of blood due to invagination of the superficial ovarian coelomic epithelium with subsequent metaplasia in typical glandular epithelium and stroma has been described by Nisolle and Donnez (1). It sometimes may result impossible to think that an endometrioma may develop from a functional structure such as a corpus luteum, however it is an implication to be considered. Somigliana et al. confirmed that ovulation may be considered a critical process involved in the origin of endometriomas and studied the management of endometriosis in the infertile patient (2).

The present case shows the transition from a hemorrhagic corpus luteum to an endometrioma as demonstrated by ultrasound images and by histology. High Doppler resistive indices of the cyst flow suggest an eventual explanation for the persistence and transformation of the hemorrhagic corpus luteum to endometrioma.

\section{CASE RePOrT}

A 38 year old patient, G0 P0, with no sexual intercourses referred, was sent to our Institution for a clinical examination after long periods, even months without menses and was sent to our Institution two weeks before a mild blood spotting.

Transabdominal ultrasound pelvic examination performed in $8^{\circ}$ day of menses revealed an endometrial pattern (linear) not in agree with the menstrual period, while nothing abnormal was seen in the adnexa.

Three weeks later the patient complained intense pelvic pain in the right iliac fossa and intense tenesmus. The transabdominal examination revealed a hypoechoic fluid cyst of 15 x $26 \mathrm{~mm}$ in the right ovary, with the ultrasound patterns of a hemorrhagic corpus luteum cyst (internally a predominant hypoechogenic pattern with linear hyperechogenic lines in the absence of color flow as assessed by Doppler technology), with the classic "ring of fire" of the corpus luteum. Resistive indices were surprisingly high (RI 0.78 and PI 1.84) comparing with the normal trend of a corpus luteum vasculature indices. 
Although the patient did not present menses afterwards, a control was performed 4 weeks later because of persistent pain and tenesmus and at that time the cyst was found to be bigger $(46 \times 55 \mathrm{~mm})$ with respect to the first examination, and classical changes as expected in hemorraghic corpus luteym were not observed (persistence of hyperechogenic lines and ovarian resistances persisting high).

Menses returned after 8 weeks and another examination was performed in the 5th day and the cyst presented dimensions somewhat reduced $(42 \times 62 \mathrm{~mm})$, the ultrasound pattern was similar to all other ultrasound examinations and ovarían resistances kept high.

Due to a continuous pelvic pain with no relief to antinflammatory oral therapy, the patient underwent a laparoscopy. A chocolate cyst was found in the right ovary and it was removed. Histology showed and confirmed the presence of an endometrioma.

\section{CONCLUSION}

Vercellini et al (3) demonstrated by serial ultrasonographic scans, a direct transition form a hemorrhagic cystic corpus luteum to an endometriotic cyst. They concluded that bleeding from a corpus luteum seems to be a critical event in the development of endometriomas.

Vascular resistance within the corpus luteum is modeled as a relatively invariable parameter, fixed at a low level by the morphological characteristics of the luteal vasculature. Studies have shown that angiogenesis actively occurs during the early luteal phase and is completed by the mid-luteal phase. Conversely, in the late luteal phase there is a process of structural luteolysis in the corpus luteum mediated by blood vessel regression (4).

In case of endometriotic cysts, resistive indices are shown to be higher than those in the corpus luteum or corpus luteum cysts with RI ranging from 0.56 to 0.67 in different study groups (5).

The possible change of a hemorragic corpus luteum cyst in an endometrioma, should be taken in mind, if not disappearing spontaneously in time and presenting persistent high ovarian resistances.

Doppler ovarían resistances evaluation of the hemorragic corpus luteum cysts is suggested in order to predict the successive monitoring, because if high, may be susceptible to a probable transformation in endometriomas.

\section{REFERENCES}

[1] Nisolle M, Donnez J. Peritoneal endometriosis, ovarian endometriosis, and adenomyotic nodules of the rectovaginal septum are three different entities. Fertil Steril. 1997; 68: 58596.

[2] Somigliana E, Vigano P, Benaglia L, et al. Management of Endometriosis in the Infertile Patient. Semin Reprod Med. 2017; 35: 31-37.

[3] Vercellini P, Somigliana E, Vigano $P$, et al. Blood on the tracks from corpora lutea to endometriomas. BJOG. 2009; 116: 366-71.

[4] Sugino N, Matsuoka A, Taniguchi K, et al: Angiogenesis in the human corpus luteum. Reprod Med Biol. 2008, 7: 91-103

[5] Kupesic S, Kurjak A. The assessment of normal and abnormal luteal function by transvaginal color Doppler sonography. Eur J Obstet Gynecol Reprod Biol. 1997; 72: 83-7.

Citation: Juan Piazze, Ettore Palma, Francesco Recchia, Cristina Galligani, Silvio Rea. It May Happen: Endometrioma from Hemorrhagic Corpus Luteum. ARC Journal of Gynecology and Obstetrics 2017; 2(3):11-12. DOI: dx.doi.org/10.20431/2456-0561.0203003

Copyright: (C) 2017 Authors. This is an open-access article distributed under the terms of the Creative Commons Attribution License, which permits unrestricted use, distribution, and reproduction in any medium, provided the original author and source are credited. 